The Extragalactic Infrared Background and its Cosmological Implications

IAU Symposium, Vol. 204, 2001

Martin Harwit and Michael G. Hauser, eds.

\title{
The Planck Mission
}

\section{J. A. Tauber (on behalf of the Planck Scientific Collaboration).}

Astrophysics Division, Space Science Department of the European Space Agency, ESTEC, P.O. Box 299, 2200AG Noordwijk, The Netherlands

\begin{abstract}
We present an overview of the European Space Agency's Planck mission, its scientific objectives, and the key elements of its technical design. The current programmatic status of Planck within ESA's Scientific Programme, implementation plans, and near-term milestones, are also addressed.
\end{abstract}

\section{Overview}

\subsection{Science}

Planck (http://astro.estec.esa.nl/Planck) is a space observatory designed to image the temperature anisotropies of the Cosmic Microwave Background (CMB) over the whole sky, with unprecedented sensitivity $\left(\Delta T / T \sim 2 \times 10^{-6}\right)$ and angular resolution (better than 10 arcminutes). Planck will provide a major source of information relevant to several cosmological and astrophysical issues, such as testing theories of the early universe and the origin of cosmic structure (Tauber 1998).

The ability to measure to high accuracy the angular power spectrum of the CMB fluctuations will allow the determination of fundamental cosmological parameters such as the density parameter $\left(\Omega_{0}\right)$, and the Hubble constant $\mathrm{H}_{0}$, with an uncertainty of order a few percent (e.g., Bersanelli et al. 1996, Bond, Efstathiou, \& Tegmark 1997; Efstathiou \& Bond 1999). Planck will not only measure the temperature fluctuations of the $\mathrm{CMB}$, but also their polarisation. This measurement (e.g., Seljak 1997) will not only yield new scientific results, but will also help to analyze the CMB temperature anisotropies (in particular by resolving degeneracies in the estimation of some cosmological parameters, Zaldarriaga et al. 1997; Kamionkowski \& Kosowsky 1998).

In addition to the main cosmological goals of the mission, the Planck sky survey will be used to study in detail the very sources of emission which "contaminate" the signal due to the CMB, and will result in a wealth of information on the properties of extragalactic sources, and on the dust and gas in our own galaxy (e.g., De Zotti et al. 1999). One specific notable result will be the measurement of the Sunyaev-Zeldovich effect in many thousands of galaxy clusters (Aghanim et al. 1997; Pointecouteau et al. 1998). 


\subsection{Technical}

The scientific prescription which will allow Planck to meet its ambitious objectives calls for:

- an offset telescope with a physical aperture of $\sim 1.5$ metre to achieve the angular resolution,

- state-of-the-art broadband detectors covering the range $\sim 30$ to $\sim 1000 \mathrm{GHz}$, to achieve the required sensitivity and the ability to remove foreground sources of emission,

- a survey with all-sky coverage carried out from a far-Earth orbit, and

- extreme attention to rejection of unwanted systematic effects.

To achieve this prescription a payload was conceived for Planck (Bersanelli et al. 1996) consisting of three basic components: (1) a telescope and baffling system, providing the angular resolution and rejection of straylight, (2) a Low Frequency Instrument (or LFI) - an array of tuned radio receivers, based on HEMT amplifiers, covering the frequency range $25-110 \mathrm{GHz}$, and operated at a temperature of $20 \mathrm{~K}$, and (3) a High Frequency Instrument (or HFI), consisting of an array of bolometers operated at $0.1 \mathrm{~K}$ and covering the frequency range $90-1000 \mathrm{GHz}$.

Figure 1 shows schematically the configuration of the model payload, with the LFI and HFI sharing the focal plane formed by the telescope. The LFI horns are situated in a ring around the HFI, this being the best configuration considering the thermal, mechanical, and optical requirements of the instruments.

\subsection{Programmatics}

Planck is the third Medium-Sized Mission (M3) of ESA's Horizon 2000 Scientific Programme (http://sci.esa.int/categories/scienceprograms/). Planck was formerly called COBRAS/SAMBA. After the mission was selected and approved (in late 1996), it was renamed in honor of the German scientist Max Planck (1858-1947), Nobel Prize for Physics in 1918.

Planck is a survey-type project which is being developed and operated as a PI mission. The Announcement of Opportunity for Planck instruments was released by ESA in October 1997, and in response two proposals were received describing two instruments with very similar characteristics to those of the model payload. The proposal for a Low Frequency Instrument was led by N. Mandolesi (Istituto TeSRE - CNR, Bologna), and that for a High Frequency Instrument was led by J.-L. Puget (Institut d'Astrophysique Spatiale, Orsay). After detailed review by a committee of independent scientists, both proposals were formally accepted in February 1999. Both instruments are currently in a phase of detailed design.

The Planck reflectors will be provided via a bilateral agreement between ESA and Denmark by a consortium of Danish institutes led by H. U. NorgaardNielsen of the Danish Space Research Institute (Copenhagen).

An Invitation to Tender was issued by ESA in September 2000 to industry to present proposals for the design, construction, and launch of the Planck 


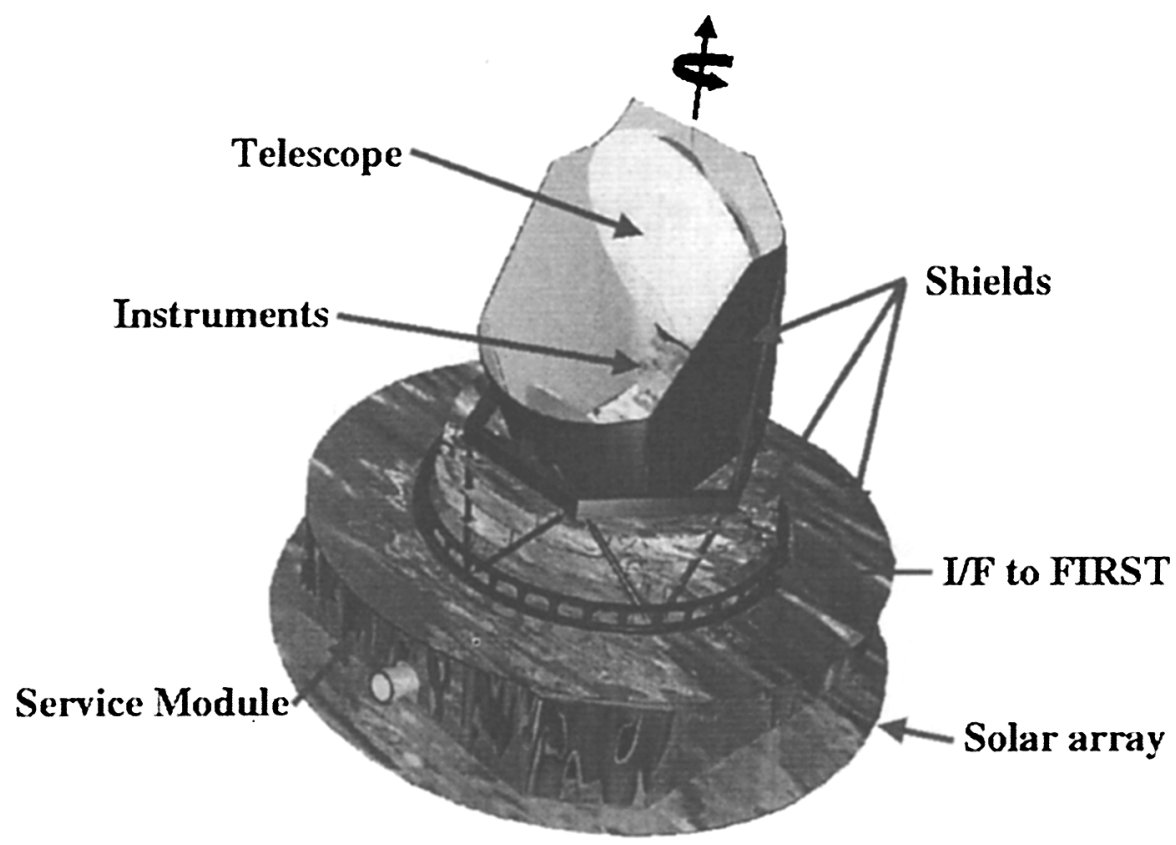

Figure 1. The Figure shows a conceptual view of the arrangement of the main elements of the Planck payload. The instrument focal plane unit contains both LFI and HFI detectors. The function of the large shield surrounding the telescope is to control the far sidelobe level of the radiation pattern as seen from the detectors. Specular cones thermally decouple the Service Module (located below the payload) from the Payload Module, and allow the payload environment to reach its nominal temperature of $\sim 50 \mathrm{~K}$. A ring located on the Service Module constitutes the interface on which FIRST is attached during launch.

spacecraft (Phases B/C/D). Phase B (detailed design) will begin in mid-2001, leading to the launch of Planck in the first quarter of 2007.

Current plans envisage the launch of Planck together with ESA's FIRST mission (Far-Infrared and Submillimetre Telescope) ${ }^{1}$. In this concept (which is often referred to as the "Carrier" because of the launch configuration, in which Planck supports FIRST above it - see Figure 2), both Planck and FIRST will be placed in orbits (of different amplitude) around the second Lagrangian point of the Earth-Sun system. 


\section{Scientific Performance}

The principal objective of Planck is to produce maps of the whole sky in ten frequency channels. The currently foreseen characteristics of the two Planck instruments (see Figure 3) are summarized in Table 1; these characteristics largely drive the quality of the final maps.
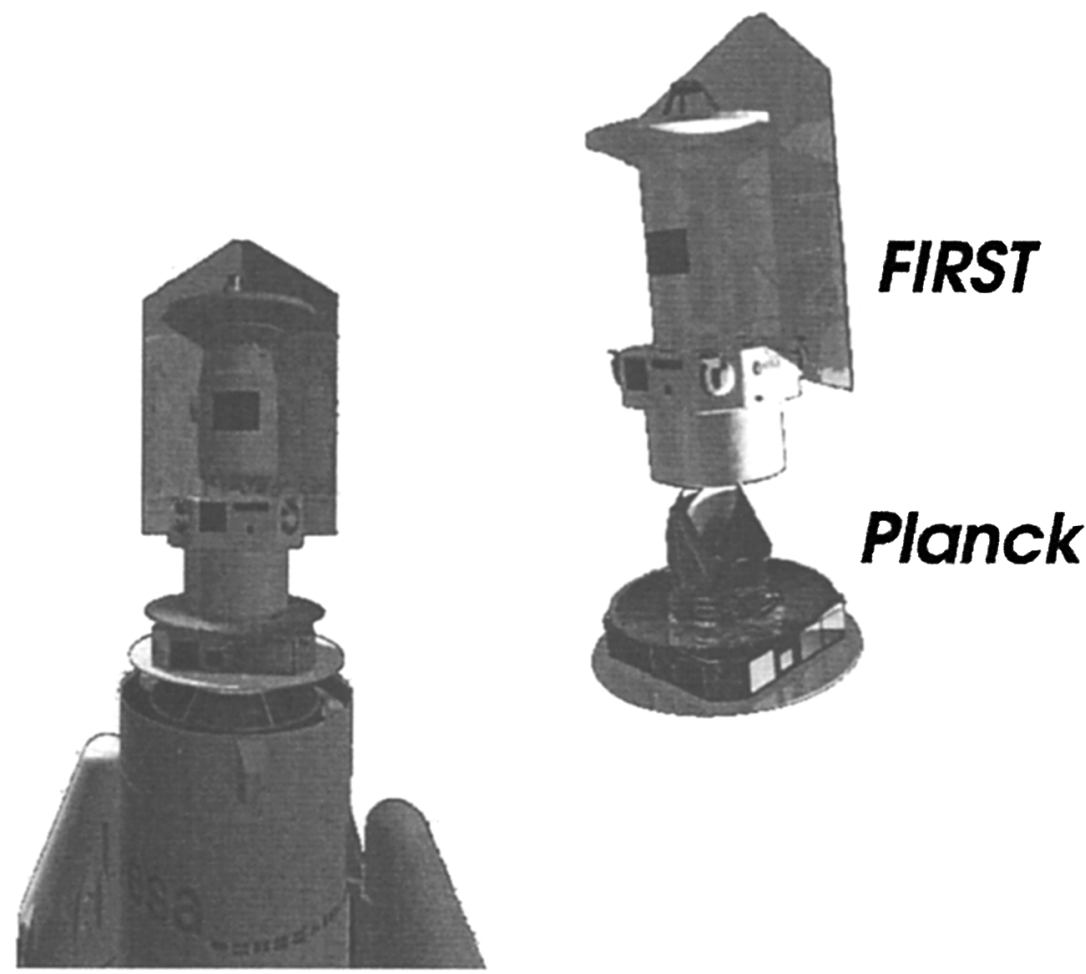

Figure 2. The Figure shows sketches of Planck and FIRST mated on the Ariane 5 launcher (fairing not shown), and the separation of the two spacecraft shortly after launch. The cylindrical mating adaptor remains attached to FIRST after separation.

These maps will not only include the CMB itself, but also all other astrophysical foregrounds, whether Galactic (free-free, synchrotron or dust) or extragalactic in origin. All ten Planck sky maps will be used to produce a single map of the cosmic microwave background anisotropies. The key that allows the mission to reach this objective is the wide spectral coverage achieved by Planck. Each astrophysical foreground has a distinct (albeit at present poorly known) spectral characteristic. Specialized data processing algorithms (e.g., Tegmark 1997; Hobson et al. 1998; Bouchet and Gispert 1999) will use this information to iteratively extract the signal due to each foreground component, until only the CMB signal remains. Instrumental systematic effects (e.g., Delabrouille 1998; Burigana et al. 1998; Maino et al. 1999; Wu et al. 2000), as well as uncertainties 
Table 1. The currently foreseen characteristics of the Planck payload.

\begin{tabular}{|c|c|c|c|c|c|c|c|c|c|c|}
\hline Telescope & \multicolumn{10}{|c|}{$\begin{array}{l}\text { Offset, } 1.5 \mathrm{~m} \text {. (proj. apert.), Temp. } \sim 50 \mathrm{~K}, \boldsymbol{\epsilon}_{\text {system }} \sim 1 \% \\
\text { Shared focal plane, viewing direction offset } 85^{\circ} \text { from spin axis }\end{array}$} \\
\hline Instrument & \multicolumn{4}{|c|}{ LFI } & \multicolumn{6}{|c|}{ HFI } \\
\hline Center Frequency (GHz) & 30 & 44 & 70 & 100 & 100 & 143 & 217 & 353 & 545 & 857 \\
\hline Detector Technology & \multicolumn{4}{|c|}{ HEMT radio rx arrays } & \multicolumn{6}{|c|}{ Bolometer arrays } \\
\hline Detector Temperature & \multicolumn{4}{|c|}{$\sim 20 \mathrm{~K}$} & \multicolumn{6}{|c|}{$0.1 \mathrm{~K}$} \\
\hline Cooling Requ & \multicolumn{4}{|c|}{$\mathrm{H}_{2}$ sorption cooler } & \multicolumn{6}{|c|}{$\mathrm{H}_{2}$ sorp. $+4 \mathrm{~K}$ J-T stage + Dilution } \\
\hline Number of Detectors & 4 & 6 & 12 & 34 & 4 & 12 & 12 & 6 & 8 & 6 \\
\hline Bandwidth $(\Delta \nu / \nu)$ & 0.2 & 0.2 & 0.2 & 0.2 & 0.25 & 0.25 & 0.25 & 0.25 & 0.25 & 0.25 \\
\hline Angular Resolution (arcmin & 33 & 23 & 14 & 10 & 10.7 & 8.0 & 5.5 & 5.0 & 5.0 & 5.0 \\
\hline $\begin{array}{l}\text { Average } \Delta T / T \text { per pixel } \\
\left(12 \text { mos., } 1 \sigma, 10^{-6} \text { units }\right)\end{array}$ & 1.6 & 2.4 & 3.6 & 4.3 & 1.7 & 2.0 & 4.3 & 14.4 & 147.0 & 6670 \\
\hline Sensitive to linear pol. & yes & yes & yes & yes & no & yes & yes & no & yes & no \\
\hline
\end{tabular}

in the recovery of parameters characterizing the foregrounds will degrade the final fidelity of the CMB maps.

Therefore, the final scientific performance of the mission depends not only on the instrumental behavior, but also on the detailed nature of the various astrophysical foregrounds, the behavior of many systematic effects which produce spurious signals (such as straylight), and the ability to remove these signals from the measured data by means of data processing algorithms. Current estimates of the performance of Planck are based on simulations of the measurement process (e.g., Bersanelli et al. 1996; Bouchet \& Gispert 1999; Knox 1999; Tegmark et al. 2000), which include such effects, as well as consideration of the signal extraction process - all to the best of available knowledge. These simulations suggest that the ability to extract the CMB signal from the measurements will be limited mainly by the background of noise originating in unresolved structure in the various foregrounds.

The Planck instruments are also designed to provide information on the polarisation state of the CMB. Although simulations of the extraction of polarisation from Planck are at a less sophisticated level than those dealing with temperature anisotropies, it is expected that Planck will be able to measure with good accuracy the angular power spectrum of the "E-component" of CMB polarisation ( $\mathrm{Hu} \&$ White 1997), with a consequent improved estimate of cosmological parameters (Bouchet et al. 1999; Prunet et al. 2000).

Naturally, as a by-product of the extraction of the CMB, Planck will also yield all-sky maps of all the major sources of microwave to far-infrared emission, opening a broad expanse of astrophysical topics to scrutiny. In particular, the physics of dust at long wavelengths and the relative distribution of interstellar matter (neutral and ionized) and magnetic fields will be investigated using dust, free-free and synchrotron maps. In the field of star formation, Planck will provide a systematic search of the sky for dense, cold condensations which are the first stage in the star formation process. One specific and local distortion of the CMB which will be mapped by Planck is the Sunyaev-Zeldovich (SZ) effect arising from the Compton interaction of CMB photons with the hot gas of clusters of galaxies. The very well defined spectral shape of the SZ effect allows it to be cleanly separated from the primordial anisotropy. The physics of gas condensation in 
cluster-size potential wells is an important element in the quest to understand the physics of structure formation and ultimately of galaxy formation.

Therefore, even though that is not its primary scientific objective, Planck will deliver high quality all-sky maps of all extended foreground emission components between centimetre and submillimetre wavelengths. These maps will constitute a scientific product which is comparable to the IRAS and COBEDIRBE maps at shorter wavelengths. Some of the particular characteristics that will make these products an invaluable resource, and that are driven by the need to image the anisotropies of the CMB, are:

- They will provide all-sky coverage with high angular resolution, varying from $\sim 30^{\prime}$ at centimetre wavelengths to $\sim 5^{\prime}$ in the far-infrared, and a per-pixel sensitivity of order microKelvins over most of the frequency range covered. In addition, due to the specific observing strategy of Planck, small regions of the sky will be observed for much longer times than average, and offer the potential of highly enhanced sensitivities (depending on the detailed behavior of systematic effects).

- They will provide an unprecendented broad-band (30 to $900 \mathrm{GHz}$ ) view of the physical processes of emission in our and other galaxies.

- They will be extremely well calibrated. Three elements contribute to this fact:

- The angular responsivity of each detector will be accurately mapped to high dynamic range (as high as $100 \mathrm{~dB}$ ), using point-like sources which travel through the beams at all times (outer planets at nearboresight angles, and the Sun, Moon, Earth and the Milky Way itself at mid- and far-boresight angles). Maps of all beam patterns over $4 \pi$ will be a necessary by-product of the process of removal of straylight and other spurious signals, and are only made possible by the all-sky nature of the survey.

- Planck is designed and operated to avoid systematic effects and allow the recovery of the CMB signal at microKelvin levels, in particular the far-Earth orbit, and extremely stable environment in the permanent shadow of the Sun will suppress such effects to the lowest possible level.

- Although Planck will not carry on-board black-body calibrators (since its objective is not to measure the average temperature of the CMB, but only deviations from it), it will be able to use previous highly accurate determinations of celestial phenomena to obtain continuous calibration information. Such phenomena include the temperature of the CMB (measured by COBE/FIRAS to $\sim 0.1 \%$ accuracy Mather et al. 1999), the amplitude of the CMB dipole (measured to $\sim 20 \mu \mathrm{K}$ by COBE/DMR - Bennett et al. 1996), the seasonal variation of the dipole (accurately known from fundamental constants), and measurements of the emission of the Milky Way (calibrated to $\sim 1 \%$ by COBE/FIRAS). The goal of Planck is to obtain a photometric calibration of order $1 \%$ or better at all frequencies below $\sim 400 \mathrm{GHz}$ and $3 \%$ above. 

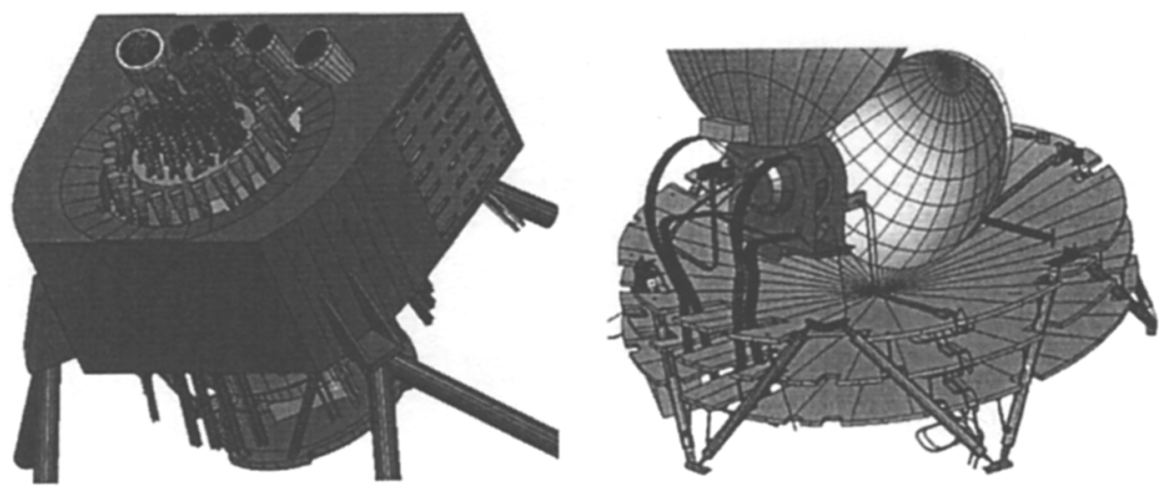

Figure 3. The Figure at left shows the combined focal plane unit formed by the LFI and HFI. The HFI unit is inserted into the ring formed by the LFI horns, and includes thermal stages at $20 \mathrm{~K}, 4 \mathrm{~K}$, $2 \mathrm{~K}$ and $0.1 \mathrm{~K}$. The sizes of the various horns correspond to different frequency channels. The location of the focal plane unit with respect to the telescope is shown at right. The cold LFI unit $(20 \mathrm{~K})$ is attached by waveguides (black in the Figure) to a warm back-end unit (300 K) located in the Service Module.

These characteristics of the Planck products will present a unique opportunity to explore in a global sense all the extended emission components of our Galaxy as well as external galaxies.

\section{Mission profile}

Planck will be launched together with FIRST (Figure 2) in the first quarter of 2007 by an Ariane 5 rocket from the European spaceport in Kourou (French Guiana). Planck and FIRST will separate immediately after launch, and each will proceed independently to different orbits around the L2 point of the EarthSun system. At this location, the payload can be continuously pointed in the anti-Sun direction, thus minimizing potentially confusing signals due to thermal fluctuations and straylight entering the detectors through the far sidelobes.

The transit time for Planck will be of order 3 months. This period will be used for commissioning and performance verification of the spacecraft and instruments. The spacecraft (S/C) will be placed into a Lissajous orbit around L2 characterised by a $\sim 6$ month period and a maximum elongation from L2 of about $280000 \mathrm{~km}$, such that the Sun-S/C-Earth angle will not exceed $10^{\circ}$. From this orbit, Planck will carry out two complete surveys of the full sky, for which it requires between 12 and 14 months of observing time.

The relevant elements of the orbit and payload orientation are shown schematically in Figure 4.

The satellite will rotate at $1 \mathrm{rpm}$ around a spin axis pointed within $10^{\circ}$ of the Sun. The payload will always remain in the shadow of the Sun. The solar array ensures this as long as it is inclined with respect to the Sun-S/C line by less than $10^{\circ}$. The Planck telescope defines a sparsely sampled field of view 


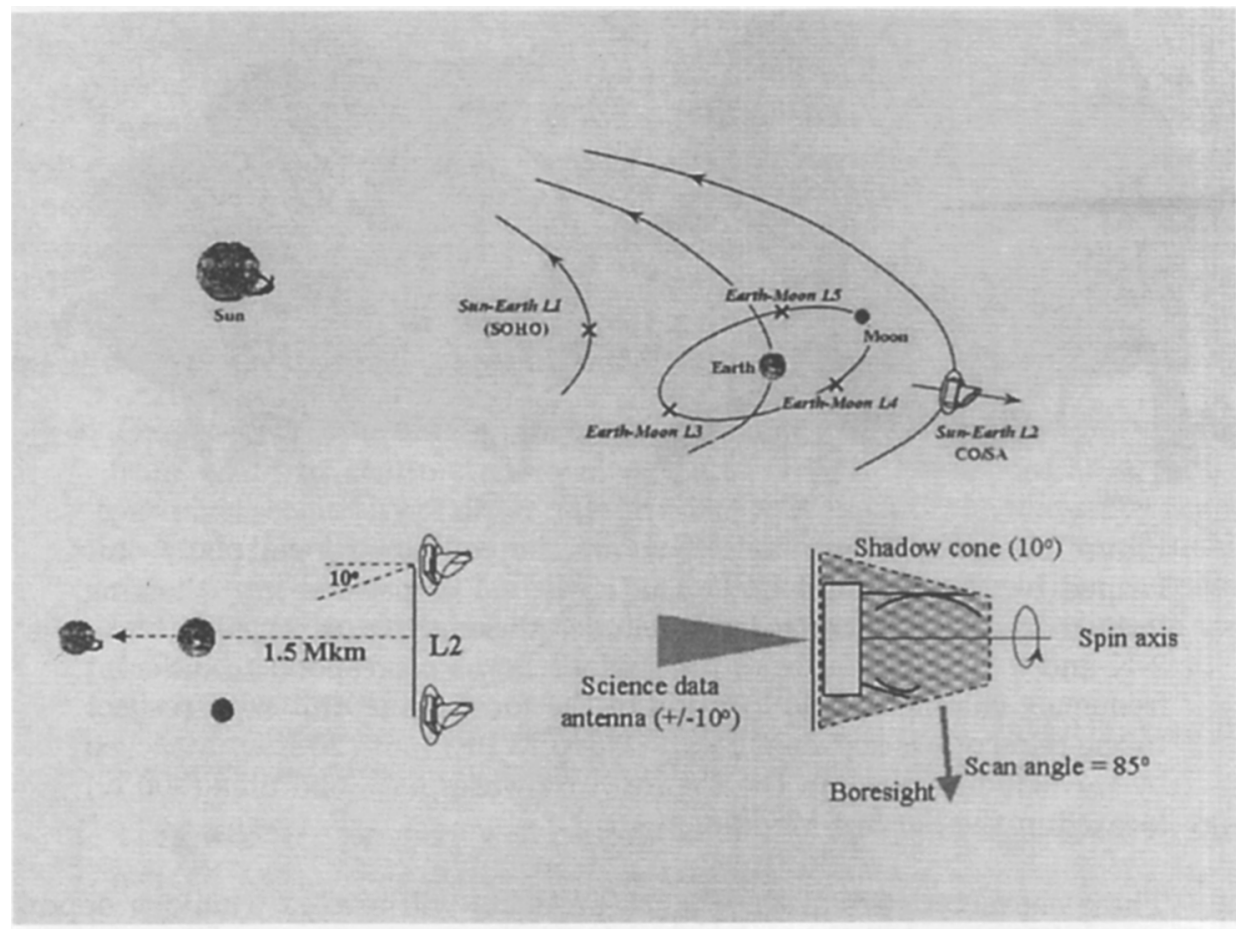

Figure 4. The baseline orbit chosen for Planck is a Lissajous orbit around the L2 point of the Earth-Sun system. A sketch of the spacecraft outlines the main constraints related to payload shadowing and telecommunications.

(FOV) approximately $8^{\circ}$ in diametre around a reference line-of-sight which is inclined by $85^{\circ}$ with respect to the spin axis. As the satellite rotates, the FOV will thus trace a circle of diametre $170^{\circ}$ on the sky.

In order to carry out its two consecutive full-sky surveys and maintain the payload in the solar shadow, the spin axis of Planck must be displaced on the average by $1^{\circ}$ per day in the direction defined by the orbital motion of the Earth around the Sun. This is achieved by spin axis depointing manoeuvers at regular intervals. As the spin axis is displaced, the observed circle also moves and gradually covers a large fraction of the sky.

The simplest possible scanning law consists of regular manoeuvers to maintain the spin axis aligned with the Sun-S/C direction, e.g., an hourly manoeuver of amplitude 2.5 arcminutes along the ecliptic plane. This scanning law results in less than full coverage of the sky, as two $10^{\circ}$ diametre polar caps will remain unobserved for each detector within the FOV (the unobserved areas will be different for each detector depending on its location within the FOV). The sky coverage achieved by Planck may be increased by tilting the spin axis away from the ecliptic plane (within the limits allowed by the solar shadow cone and the telemetry antenna), thus allowing many detectors to observe the ecliptic poles. 
It is important for Planck to be able to remove systematic effects (e.g., instrumental drifts) which contaminate the observations. To achieve this it is necessary to maintain a high level of redundancy, i.e., that a given location of the sky be observed many times (both with short and with long time scale periodicity), with different detectors and with different satellite attitudes. In this respect it may be useful to implement a scanning law which results in each ring crossing many other rings at a range of locations along it (e.g., Delabrouille 1998; Revenu et al. 2000). Given the payload configuration, these crossings will be clustered at high ecliptic latitudes. However, by tilting the spin axis with respect to the ecliptic plane, the distribution of crossings may be spread over a larger range of ecliptic latitudes.

Therefore, both to increase its sky coverage, and to increase the redundancy over a wider range of ecliptic latitudes, it is likely that the scanning law implemented by Planck will deviate significantly from the most basic one described above. The specific scanning law to be used will be established by means of detailed simulations during the development of the mission, and may also be modified by tests carried out in flight during the Performance Verification phase. It is also possible that the scanning law used during the second sky survey differs significantly from that used in the first survey.

Planck will dump each day to Earth within a period of 2 hours the data acquired during 24 hours. Observations will not be interrupted during the downlink period, and the S/C will not be reoriented towards the Earth. Therefore the telemetry antenna is designed to have adequate gain within a $10^{\circ}$ half-cone from the spin axis, ensuring that even at the extremes of its orbit the Planck telemetry can achieve full bandwidth.

\section{Operations and Data Processing}

The Planck spacecraft will be controlled from a dedicated Mission Operations Centre developed and operated by ESOC in Darmstadt (Germany). From there, the scientific data produced by Planck will be piped daily to two Data Processing Centres (DPCs), which will be developed and operated by the two Consortia selected to provide the Planck instruments.

In particular, the two DPCs will be responsible for:

- operating and calibrating their respective instruments,

- daily and long term analysis of instrument health and performance,

- daily analysis of science data, and

- all levels of processing of Planck data, from raw telemetry to deliverable scientific products.

The two DPCs will share a basic information management infrastructure, the Planck Integrated Data and Information System (IDIS, Bennett et al. 2000). IDIS is being conceived from an object-oriented point of view, and is planned to contain five different components:

- a Document Management Component, containing all relevant documentation, 
- a Software Management Component, encompassing the software in common between the two Consortia,

- a Process Coordinator Component, providing a single software environment for data processing (e.g., a data pipeline manager),

- a Data Management Component, allowing the ingestion, efficient management, and extraction of the data (or subsets thereof) produced by Planck activities,

- a Federation layer, providing inter-connection among IDIS components (e.g., relating objects controlled by each component).

The main scientific products of the mission will be produced by the two DPCs jointly, and will consist of all-sky maps in ten frequency bands, which will be made publicly available one year after completion of the mission, together with a first generation set of maps of the CMB, Sunyaev-Zeldovich effect, Galactic emission (dust, free-free, and synchrotron), and point-source catalogs. The time series of observations (after calibration and position reconstruction) will also eventually be made available as an on-line archive.

\section{Acknowledgments}

Planck (http://astro.estec.esa.nl/Planck) is an ESA project with instruments funded by ESA member states (in particular the PI countries: France and Italy), and with special contributions from Denmark and NASA (USA). This paper draws widely from the work carried out in the past years by the Planck Instrument and Reflector Provider Consortia, ESA, ESOC, and industry (principally the former Matra Marconi Toulouse, and Alcatel Space Cannes).

\section{References}

Aghanim, N., de Luca, A., Bouchet, F. R., Gispert, R., \& Puget, J.-L. 1997, A\&A, 325, 9

Bennett, C. L., Banday, A. J., Gorski, K. M., Hinshaw, G., et al. 1996, ApJ, 464, L1

Bennett, K., Pasian, F., Sygnet, J., Banday, A., et al. 2000, Proc. SPIE Vol. 4011, Advanced Global Communications Technologies for Astronomy, ed. R. I. Kibrick \& A. Wallander, 2

Bersanelli, M., et. al. 1996, Report on the Phase A Study of COBRAS/SAMBA, ESA D/SCI $(96) 3$

Bond, J. R., Efstathiou, G., \& Tegmark, M. 1997, MNRAS, 291, L33

Bouchet, F., \& Gispert, R. 1999, New Astronomy, 4, 443

Bouchet, F. R., Prunet, S., \& Sethi, S. K. 1999, MNRAS, 302, 663

Burigana, C., Maino, D., Mandolesi, N., Pierpaoli, E., et al. 1998, A\&AS, 130, 551

Delabrouille, J. 1998, A\&AS, 127, 555 
de Zotti, G., Toffolatti, L., Argeso, F., Davies, R. D., et al. 1999, in AIP Conf. Proceedings, 476, 3K Cosmology, ed. L. Maiani, F. Melchiorri, \& N. Vittorio (Woodbury, N.Y.: AIP), 204

Efstathiou, G., \& Bond, J. R. 1999, MNRAS, 304, 75

Hobson, M. P., Jones, A. W., Lasenby, A. N., \& Bouchet, F. R. 1998, MNRAS, 300,1

Hu, W., \& White, M. 1997, New Astronomy, 2, 323

Kamionkowski, M., \& Kosowsky, A. 1998, Phys. Rev. D, 57, 685

Knox, L. 1999, MNRAS, 307, 977

Maino, D., Burigana, C., Maltoni, M., Wandelt, B. D., et al. 1999, A\&AS, 140, 383

Mather, J., Fixsen, D. J., Shafer, R. A., Mosier, C., Wilkinson, D. T. 1999, ApJ, 512,511

Pointecouteau, E., Giard, M., Barret, D. 1998, A\&A, 336, 44

Prunet, S., Sethi, S. K., \& Bouchet, F. R. 2000, MNRAS, 314, 348

Revenu, B., Kim, A., Ansari, R., Couchot, F., et al. 2000, A\&AS, 142, 499

Seljak, U. 1997, ApJ, 482, 6

Tauber, J. 1998, in Proceedings of the XXIIIrd Moriond Symposium, Fundamental Parameters in Cosmology

Tegmark, M. 1997, ApJ, 480, L87

Tegmark, M., Eisenstein, D. J., Hu, W., \& De Oliveira-Costa, A. 2000, ApJ, 530,133

$\mathrm{Wu}$, J. H. P., et al. 2000, astro-ph/0007212

Zaldarriaga, M., Spergel, D. N., \& Seljak, U. 1997, ApJ, 488, 1 